\title{
Du cabinet de curiosités au musée scientifique. Le musée italien et la genèse des musées à Montréal dans la première moitié du XIX ${ }^{\mathrm{e}}$ siècle
}

\section{Hervé Gagnon}

Volume 45, numéro 3, hiver 1992

URI : https://id.erudit.org/iderudit/304993ar

DOI : https://doi.org/10.7202/304993ar

Aller au sommaire du numéro

Éditeur(s)

Institut d'histoire de l'Amérique française

ISSN

0035-2357 (imprimé)

1492-1383 (numérique)

Découvrir la revue

Citer cette note

Gagnon, H. (1992). Du cabinet de curiosités au musée scientifique. Le musée italien et la genèse des musées à Montréal dans la première moitié du XIX ${ }^{\mathrm{e}}$ siècle. Revue d'histoire de l'Amérique française, 45(3), 415-430.

https://doi.org/10.7202/304993ar 


\title{
DU CABINET DE CURIOSITÉS AU MUSÉE SCIENTIFIQUE LE MUSÉE ITALIEN ET LA GENÈSE DES MUSÉES À MONTRÉAL DANS LA PREMIÈRE MOITIÉ DU XIX ${ }^{\mathrm{e}}$ SIÈCLE ${ }^{1}$
}

\author{
HERVÉ GAGNON \\ Agent de recherche \\ Programme de maîtrise en muséologie \\ Université de Montréal
}

Le cabinet de curiosités constitue un élément marquant de l'histoire des musées. Caractéristique du dynamique questionnement scientifique de la fin du moyen âge et des deux premiers siècles de l'époque moderne, le cabinet, créé par et pour les membres de l'élite intellectuelle, abrite à compter du $\mathrm{XV}^{\mathrm{e}}$ siècle un véritable microcosme, un abrégé de l'univers connu, voué à l'étude et à l'accumulation des connaissances. Humanistes et savants y regroupent pêle-mêle des antiquités, des monnaies, des œuvres d'art et, à partir du XVI ${ }^{e}$ siècle, des spécimens naturels et des curiosités «naturelles», «artificielles» et exotiques diverses ayant en commun la rareté et l'intérêt curieux ${ }^{2}$. L'apparition des premiers cabinets aux États-Unis à la fin du XVIII ${ }^{e}$ siècle modifie considérablement ce modèle. Le cabinet américain, abritant lui aussi des collections disparates, ajoute en effet à la vocation scientifique première une dimension éducative qui constitue sa pierre angulaire. Reposant sur une approche ludique qui implique l'accès aux collections d'un public que l'on cherche désormais à attirer, il promet au visiteur de spectaculaires curiosités qui en viennent rapidement à prendre le pas sur les habituels spécimens d'histoire naturelle, antiquités et objets d'art, donnant naissance à une curiosité-

1 Ces recherches ont été réalisées grâce à une subvention du Conseil de recherches en sciences humaines du Canada dans le cadre d'un projet intitulé «L'idée de musée au Québec (1800-1990): la collection», dirigé par Jean Trudel, responsable du programme de maîtrise en muséologie à l'Université de Montréal. L'auteur tient à remercier John A. Dickinson, Jean Trudel et Jean-Marc Larrue, qui ont bien voulu commenter une première version de cet article.

2 Krizsztof Pomian, Collectionneurs, amateurs et curieux. Paris-Venise, XVII'-XVIII siècles (Paris, Gallimard, 1987), 62-80, et «Musées et patrimoine», Patrimoine en folie (Paris, Éditions de la Maison des sciences de l'Homme, 1990), 183-184; Jean Trudel, «L'imaginaire comme objet de curiosité», Parachute, 57, 4-9.

RHAF, vol. 45, n 3, hiver 1992 
spectacle qui marquera les formes muséales durant la première moitié du XIX ${ }^{e}$ siècle $^{3}$.

Rendu familier par la renommée des cabinets américains, ce modèle de la curiosité-spectacle pénètre au Bas-Canada dès le début du XIX $\mathrm{XIè}^{\mathrm{e}}$ siè par l'intermédiaire des expositions qui font halte à Montréal et à Québec et influence fortement la conception du Musée italien, premier musée ouvert au public à Montréal en 1824 par l'aubergiste Thomas Delvechio. Entreprise autant commerciale que scientifique, ce cabinet répond aux goûts du moment et disparaît lorsque l'influence accrue des sciences dans les musées à compter de la seconde moitié du siècle en évacue la curiosité-spectacle, qui se cantonne progressivement dans les expositions itinérantes.

Liée de près à l'histoire sociale, des sciences et des mentalités, la recherche sur les musées, les collections et les expositions connaît depuis déjà plusieurs années un essor remarquable, notamment en France, aux Etats-Unis et en Grande-Bretagne. Parallèlement, depuis quelques années, l'histoire des sciences au Québec a grandement contribué à cerner la genèse et le développement des musées scientifiques. Dans l'ensemble, toutefois, l'histoire générale des musées au Canada et de leur signification sociale demeure un champ de recherche encore relativement inexploité et peu d'études ont tenté de cerner en profondeur les origines des musées québécois ${ }^{4}$. En ce sens, le

3 Voir particulièrement Edward P. Alexander, Museum Masters. Their Museums and their Influence (Nashville, American Association for State and Local History, 1983); Charles Coleman Sellers, Mr. Peale's Museum. Charles Wilson Peale and the First Museum of Natural Science and Art (New York, W. W. Norton \& Co., 1980); Walter Whitehill et al., «History of Museums in the United States», Curator, 8,1 (March 1964): 54.

4 En ce qui concerne l'Europe, mentionnons notamment, en plus de Krizsztof Pomian, op.cit., Antoine Schnapper, Le géant, la licorne, la tulipe. Collections et collectionneurs dans la France du XVII' siècle (Paris, Flammarion, 1988); Kenneth Hudson, Museums of Influence (Cambridge, Cambridge University Press, 1987); Arthur MacGregor, ed., Transcendant's Rarities. Essays on the Foundation of the Asmolean Museum, 1683 (Oxford, Clarendon Press, 1983); Oliver Impey et Arthur MacGregor, eds., The Origins of Museums. The Cabinet of Curiosities in Sixteenth and Seventeenth-Century Europe (Oxford, Clarendon Press, 1985); depuis 1988 une revue spécialisée, le Journal of the History of Collections, parait chez Oxford University Press. Pour le Québec, voir entre autres Luc Chartrand, Raymond Duchesne et Yves Gingras, Histoire des sciences au Québec (Montréal, Boréal, 1987), 481 p.; Paul Carle et Raymond Duchesne, «L'ordre des choses: cabinets et musées d'histoire naturelle au Québec (1824-1900)», Revue d' histoire de l' Amérique française, 44,1 (été 1990): 3-30. Également Paul Carle et Alain Mongeau, «Le cas de l'Université McGill et du Musée Redpath pendant la première moitié du XX' siècle», Musées, 11,1-2 (1988): 6-10, et Paul Carle, Madeleine Dufresne et Lynne Teather, «Le Musée Redpath de 1940 à 1970: les années Johannsen», ibid., 11-14. Enfin, une seule monographie tente de cerner l'histoire des musées au Canada, celle de Archie F. Key, Beyond Four Walls. The Origins and Development of Canadian Museums (Toronto, McClelland and Stewart, 1973), $384 \mathrm{p}$. Quelques études portant sur le Musée italien ont permis de poser les balises d'une réflexion plus approfondie, notamment E.-Z. Massicotte, «Noms de 
Musée italien constitue un cas-type de l'enracinement de la curiositéspectacle au Bas-Canada et de sa dissociation éventuelle du milieu des musées.

\section{1 - THOMAS DELVECHIO, PROMOTEUR DE LA CURIOSITÉ À MONTRÉAL}

Aubergiste montréalais d'origine italienne, Thomas (Tomasso) Delvechio, né sur les rives du lac de Côme en 1758, figure avec son frère Pierre (Pietro), lui aussi aubergiste, parmi les premiers Italiens venus s'établir à Montréal à la fin du XVIII ${ }^{e}$ siècle. Comme toutes les premières familles italiennes - les Donegani, les Rusconi, les Bruchési, - les Delvechio s'intègrent aisément à la société canadienne $^{5}$. En janvier 1797, Thomas épouse à Lavaltrie Thérèse Chevalier, fille de l'aubergiste Michel Chevalier. Il est alors vraisemblablement aubergiste à Montréal, où, selon les journaux de l'époque, il tient dès 1791, «au courant Sainte-Marie», l'Auberge des TroisRois ${ }^{6}$.

En 1805, Thomas Delvechio acquiert au coût de 40000 livres un terrain et une maison en pierre de deux étages, situé au 4, place du Marché, où il ouvre une auberge, fort populaire durant les années 1810-18207. C'est là qu'il installera son musée. Il devient durant cette période un propriétaire immobilier relativement prospère. Dès 1818 , il offre à louer dans le Spectateur Canadien une «belle maison de pierre, à deux étages, située dans la rue Notre-Dame, $\mathrm{N}^{\circ} 40$ » et passe devant notaire plusieurs baux de location de ses propriétés immobilières ${ }^{8}$. À son décès, il laisse à son épouse un honnête patrimoine comprenant un magasin fort bien rempli de marchandises diverses, sa maison et trois autres emplacements avec maisons - dont celui du Musée italien. La valeur totale de ses biens est alors estimée à 1159 livres, 2c, 3d, à laquelle s'ajoute la somme de 361 livres, 18c, 6d en argent comptant

rues, de localités, etc., à Montréal», Bulletin de recherches historiques, 30 (1924): 175-177, et, plus récemment Raymond Duchesne, «Delvecchio, Thomas», Dictionnaire biographique du Canada, 6: 202-204, ainsi que Philippe Dubé et Raymond Montpetit, «Naissance de nos premiers musées», Cap-aux-Diamants, 25 (printemps 1991): 10-13.

5 Duchesne, op. cit., 202; Bruno Ramirez, Les premiers Italiens de Montréal. L'origine de la Petite Italie du Québec (Montréal, Boréal Express, 1984), 12.

6 Duchesne, op. cit.; La Gazette de Montréal, 15 décembre 1791.

7 ANQ-M, greffe de Louis Chaboillez, ler mars 1805, $\mathrm{n}^{\circ}$ 5060; Thomas Doige, An Alphabetical List of Merchants, Traders and Housekeepers Residing in Montreal (1819), 82; Robert Rumilly, Histoire de Montréal (Montréal, Fides, 1970), 2: 134.

8 Le Spectateur canadien, 17 octobre 1818; ANQ-M, greffe Nicolas-Benjamin Doucet, baux de location: 31 août 1822, $\mathrm{n}^{\circ} 10009 ; 7$ avril 1825, $\mathrm{n}^{\circ} 12469 ; 27$ mars 1826, $\mathrm{n}^{\circ} 13467$. 
et des dettes actives de plus de 945 livres $^{9}$. Commerçant toujours à l'affût d'une attraction rentable, Delvechio participera pleinement à la vogue de la curiosité-spectacle à Montréal et s'inspirera des cabinets de curiosités américains et des expositions itinérantes pour façonner un musée répondant au goût du public.

\section{LA CURIOSITÉ À MONTRÉAL (1817-1847)}

Le contenu des expositions qui passent par Montréal démontre clairement cet intérêt pour la curiosité-spectacle, tant au niveau des organisateurs que du public. Si l'on entend par «exposition» le fait de présenter au public un quelconque objet dans un but de récréation ou d'enrichissement des connaissances, un survol des annonces des journaux montréalais ${ }^{10}$ entre 1817 et 1847 permet de relever un total de 50 expositions dont la nature démontre clairement les formes que prend la curiosité.

Rarement conçues de façon systématique, tenues le plus souvent dans des établissements hôteliers, parfois aussi dans des salles publiques diverses ou des lieux sacrés, les expositions montréalaises de la première moitié du XIX ${ }^{\mathrm{e}}$ siècle, dont le prix d'entrée est généralement modeste, se multiplient de juillet à septembre, saison au cours de laquelle on retrouve $50 \%$ du total des expositions présentées. Les 26 annonces qui précisent la provenance des expositions nous apprennent qu'elles sont généralement d'origine étrangère, le plus souvent américaine. Elles sont toutefois soutenues par des promoteurs montréalais qui, selon les 35 annonces qui précisent la nature des promoteurs, se partagent de façon assez égale entre anglophones (17) et francophones (13), ainsi que quelques italiens (5).

De facture populaire, elles se réclament de la science tout en visant à l'exhibition du différent, de l'inhabituel, de ce qui défie les normes de la nature. Ainsi, 6 expositions (12\%) offrent au public ce qu'il est permis de qualifier de curiosités animales dans la mesure où l'animal est abordé non pas dans une perspective scientifique mais comme lusus naturae: par exemple, une brebis de Lavaltrie exposée au public en 1823 dont la tête et le visage «ont une ressemblance frappante avec la figure humaine; le reste du corps et les membres

9 ANQ-M, Testament de Thomas Delvechio, greffe Nicolas-Benjamin Doucet, 4 mai $1826, n^{\circ} 13583$; ANQ-M, Inventaire après décès de Thomas Delvechio, greffe Nicolas-Benjamin Doucet, ler février $1827, \mathrm{n}^{\circ} 14331$.

10 La Gazette de Montréal, Le Spectateur canadien, La Minerve, The Montreal Herald, la Bibliothèque canadienne, L'Aurore, Le Courrier du Bas-Canada, La Revue Canadienne et $L$ 'Aurore des Canadas, dans lesquels nous avons recensé les annonces et les critiques des expositions tenues au cours de la période. 
sont ceux d'un agneau»; «un jeune orignal d'un an, qui fait plusieurs jeux, à l'ordre de son maitre»; «une grande vache mammoth exhibée en 1830 mesurant 15 mains de hauteur, avec son veau de deux mois, qui mesure deux pieds et dix pouces de hauteur»; un bœuf Columbus montré en 1831 «pesant plus de 4000 livres (qui) a été exhibé dans les principales villes des États-Unis et a été vu par plus de cent mille personnes, qui toutes disent que c'est le plus grand bœuf qui ait jamais été exhibé, et qu'il ne peut être surpassé» ${ }^{11}$.

La curiosité prend également la forme de mécaniques diverses, essentiellement des automates, dont 5 expositions (10\%) attirent les Montréalais au cours de la période étudiée. Mentionnons notamment le panorama mécanique de $\mathrm{M}$. Jacotel, exposé en 1821 et formé de figurines automates «dont une partie forment une musique, et paraissent jouer avec une grande exactitude une variété d'airs sur différents instruments (et dont les autres) travaillent à divers ouvrages mécaniques, et agissent de la manière la plus (...) naturelle»; les «androïdes ou mécanismes animés» de M. Haddock, en 1826, dont un critique dit espérer que «tous ceux qui ont du goût pour le merveilleux, ou pour les amusements raisonnables, ainsi que ceux qui sont disposés à encourager les arts mécaniques et à favoriser le génie inventeur» iront voir le spectacle; en 1835 , le pendule à mouvement perpétuel de M. Hoffmester. Quelques «curiosités artisanales» ( 2 expositions), des objets de verre soufflé ou fondu auquel on donne des formes inhabituelles, sont également offertes à la vue du public ${ }^{12}$.

C'est toutefois dans l'exposition de «curiosités humaines» que l'on retrouve de la façon la plus marquée cet intérêt pour le morbide et le monstrueux qui constitue une partie intégrante de la mentalité curieuse. En effet, sur les 50 expositions présentées à Montréal entre 1817 et 1847, $8(16 \%)$ mettent en évidence des cas souvent pathétiques d'aberrations naturelles: un nain de 49 ans, mesurant trois pieds deux pouces et «habillé de manière comique (que) les admirateurs des effets merveilleux de la nature» sont invités à venir admirer en 1817, ou ces deux autres, qui s'exposent eux-mêmes au public en 1834 «comme ils n'ont d'autres moyens de gagner leur vie que leurs efforts individuels»; une «belle albinesse» exhibée en 1817; Calvin Edson, «squelette vivant de 58 livres (dont) rien dans son habillement ni son apparence (n'est susceptible d') alarmer les plus délicats» et M. Mailhot, géant de 6 pieds 4 pouces et 619 livres, «sans contredit

11 La Gazette canadienne, 26 mars 1823; Le Spectateur canadien, 12 janvier 1828; La Minerve, 9 août 1830 et 3 février 1831 .

12 Le Spectateur canadien, 11 août 1821 et 21 octobre 1826; La Minerve, 14 août 1834 et 13 juillet 1835; La Revue canadienne, 15 mai 1847. 
l'homme le plus merveilleux pour la taille» en 1831; à deux reprises des jumeaux siamois et enfin, en 1836, un enfant de 10 mois pesant 90 livres $^{13}$.

Les divers aspects de la curiosité forment donc un total de $42 \%$ de l'échantillon d'expositions présentées à Montréal de 1817 à 1847. Séparément, toutefois, l'histoire (20\%) et l'art fournissent le plus grand nombre d'expositions. Ces autres types d'exposition sont cependant loin de toujours échapper à la mentalité curieuse. En effet, les annonces d'expositions à connotation historique - essentiellement des panoramas souvent intégrés au spectacle d'un cirque de passage - mettent généralement tout autant en évidence ses dimensions exceptionnelles que sa valeur documentaire. Le même phénomène se reproduit en ce qui concerne les zoos ambulants, dont les 8 expositions représentent $16 \%$ du total et mettent le plus souvent en relief les caractéristiques curieuses des animaux exhibés: le "caractère furieux» d'urus africains présentés par Hedge \& Hyman en 1817; l'exotisme d'un éléphant mâle présenté en 1820, «le premier (...) qui ait jamais été importé en Amérique, (...) le plus grand et le plus ingénieux de tous les animaux», les 26 animaux exhibés en 1822, dont «les propriétaires ont fait de grandes dépenses pour se procurer ces rares curiosités naturelles des différentes parties du globe». Notons enfin le même phénomène dans la seule exposition $(2 \%)$ consacrée en 1819 à des verres d'optique présentant 8 points de vue «parmi les plus remarquables et les mieux achevés» et dans celle relative à l'astronomie (l'exposition en 1823 d'un microscope solaire «qui fait paraître les objets 100000 fois plus grands qu'ils ne le paraissent, ou ne le paraîtraient s'ils étaient visibles, à l'œil nu» ${ }^{14}$.

Seules les 9 expositions d'art semblent, en définitive, relativement exemptes de connotation curieuse. Elles ont pour but de vendre des œuvres ou de soutenir des causes charitables, rarement de s'en tenir au pur plaisir esthétique. Il en va ainsi, par exemple, de l'exposition de «peintures étrangères» flamandes et hollandaises tenue de novembre 1823 à mars 1824 au profit de l'orphelinat de Montréal et de l'exposition «d'anciens chefs-d'œuvres de sculpture» copiés des collections du Louvre et du Vatican, «sous le patronage du comité au bénéfice des incendiés» ${ }^{15}$.

13 Le Spectateur canadien, 6 janvier et 18 octobre 1817; La Minerve, 28 avril et 12 septembre 1831, 13 février 1834, 13 juillet 1835 et le 14 mars 1836; La Revue canadienne, 17 avril 1846.

14 Le Spectateur canadien, 23 août 1817; 19 juin 1819; 19 juin 1820; 23 août 1823; La Gazette canadienne, 4 septembre 1822.

15 Le Spectateur canadien, ler novembre 1823; La Revue canadienne, 27 juillet 1846. 


\section{UN CABINET DE CURIOSITÉS INSPIRÉ DU MODÈLE AMÉRICAIN}

Le modèle de la curiosité-spectacle marque donc considérablement les premières expositions à Montréal au cours de la première moitié du XIX ${ }^{\mathrm{e}}$ siècle et influence manifestement Thomas Delvechio. On le retrouve en effet très tôt dans ce monde des attractions inhabituelles alors qu'il tente d'attirer la clientèle à l'Auberge des Trois-Rois en y présentant divers numéros d'habileté et de curiosités. Dès décembre 1791, il annonce ainsi dans la Gazette de Montréal une soirée au cours de laquelle «la Compagnie du Sr. Donegani représentera une variété de Tours de Souplesse très-curieux, par le Jeune Hercules, âgé de huit ans (...); il dansera la Danse aux CEufs les yeux bandés, pour la première fois, $\&$ il fera plusieurs autres Tours qui surprendront les Spectateurs». On y présente également «une Ménagerie de Chiens qui font exactement tout ce qu'on leur commande à la parole» et qui réalisent les tours les plus variés. En 1792, il offre de nouveau ce spectacle, auquel s'ajoute «pour curiosité, une perruque d'homme, une coiffure de femme et un chapeau, le tout fait avec du verre filé» ${ }^{16}$.

C'est en 1822, à une époque où Michel Bibaud publie la Bibliothèque Canadienne ${ }^{17}$ et où les infrastructures culturelles commencent à se développer à Montréal avec l'aménagement du Théâtre Royal et de la Montreal Library, que Delvechio entreprend la création d'un cabinet de curiosités «naturelles et artificielles» à l'américaine. Fort d'un voyage «dans les principales villes des États-Unis, et y (ayant) vu ce qu'on peut y voir de plus interessant en ce genre» ${ }^{18}$, il cherche à reproduire à Montréal des cabinets tels le musée de la Salem East India Society ou de la Charleston Library Society, le Western Museum de Cincinnati et l'American Museum de New York, où se côtoient les spécimens d'histoire naturelle, les portraits d'hommes célèbres, les figures de cire, les objets ethnologiques, les reliques historiques, les aberrations de la nature et les curiosités les plus diverses. Même le Peale's American Museum de Charles Wilson Peale à Philadelphie voit sa vocation scientifique originelle subir à compter de 1820 un glissement marqué vers le spectacle ${ }^{19}$.

16 La Gazette de Montréal, 15 décembre 1791, 19 avril et 6 septembre 1792.

17 Rumilly, op. cit., 164.

18 Michel Bibaud, «Cabinet de curiosités naturelles et artificielles. On y voit réunis l'utile et l'agréable», Bibliothèque canadienne, 2,1 (juillet 1825): 53-54.

19 Edward P. Alexander, Museums in Motion. An Introduction to the History and Functions of Museums (Nashville, American Association for State and Local History, 1979), 47 49, et Museums Masters, op. cit., 45-77; Sellers, op. cit., 215-254; Whitehill et al., op. cit., 58, 17-21; Key, op. cit., 59-62; Gary Kulik, «Designing the Past: History-Museum Exhibitions from Peale to Present», Warren Leon et Roy Rosenzweig, eds., History Museums in the United States. A Critical Assessment (Chicago, University of Illinois Press, 1989), 5-6. 
Delvechio orchestre dans les journaux une vaste campagne de publicité. De novembre 1822 à janvier 1823, il fait paraître dans les journaux montréalais une publicité pour annoncer l'ouverture prochaine de son établissement et inviter le public à lui proposer des curiosités dont il pourrait faire l'acquisition:

CURIOSITÉS: Le soussigné ayant voyagé dans différents pays, à l'effet d'y recueillir toutes les curiosités qui lui paraîtrait (sic) digne (sic) d'être mises sous les yeux du public, se propose d'ouvrir sous peu un MUSEUM à la maison No.4, sur le vieux marché où il achètera tous les articles curieux, qui lui paraîtront dignes d'être placés dans sa rare collection. ${ }^{20}$

Plus d'un an après cet énoncé d'intention, période au cours de laquelle on peut penser qu'il accroît sa collection et en prépare la présentation, Delvechio annonce en février 1824 dans le Spectateur Canadien l'ouverture du Musée italien ${ }^{21}$.

\section{LES CURIOSITÉS «NATURELLES \\ ET ARTIFICIELLES»DU MUSÉE ITALIEN}

Sis au 4, place du Vieux Marché et annoncé par une enseigne «représentant une partie des Curiosités qui y sont contenues», le Musée italien (d'abord nommé Museo italiano par son propriétaire) présente l'aménagement éclectique caractéristique du cabinet de curiosités à l'américaine. Annoncé dès août 1824 comme «Musée italien et Cabinet de curiosités», il est décrit comme renfermant «une très belle Collection de choses rares et curieuses dont une très grande partie n'ont jamais été vues dans ce pays» et, rapidement, sera désigné par la population sous le vocable typique de «Cabinet de Curiosités Naturelles et Artificielles» ${ }^{22}$.

Soucieux de ne pas aliéner la clientèle, Delvechio se hâte de préciser dès sa publicité initiale que l'on «ne trouvera rien dans le Cabinet de Curiosités qui soit le moins du monde contraire aux bonnes mœurs ou à la décence; de sorte que les personnes les plus religieuses les peuvent voir sans aucun scrupule (...) il n'y sera souffert ni propos, ni comportement indécent». De même, «il ne sera pas permis de fumer dans la salle d'Exhibition». Ouvert tous les jours, sauf les dimanches et jours de fêtes, de 9 heures «jusqu'à la brune», le Musée italien est accessible aux groupes d'au moins trois personnes pour 30 sols par

20 Le Spectateur canadien, 30 novembre 1822; La Gazette canadienne, 14 décembre 1822; The Montreal Herald, 30 novembre 1822.

21 Le Spectateur canadien, 7 février 1824.

22 Le Spectateur canadien, 14 août 1824; Bibaud, op. cit., 53. 
tête et offre des réductions pour les groupes plus nombreux. Il est également ouvert le soir, à condition d'en faire la demande 48 heures à l'avance ${ }^{23}$.

Dès sa naissance, le musée est installé à l'étroit et tout ce que regrette Delvechio «c'est que l'appartement n'en soit pas plus spacieux». Toutefois, note Michel Bibaud en 1825, «on peut dire qu'il a su si bien tirer parti de l'espace à sa disposition, et arranger le tout avec tant d'ordre, de goût, et de netteté (...) qu'il n'y aurait qu'une foule trop nombreuse de spectateurs qui pût faire percevoir du défaut dont il se plaint». Comme il est de rigueur dans les cabinets de curiosités, Delvechio exploite le moindre espace disponible pour exhiber la collection et l'entassement d'objets qui en résulte est caractéristique. Même le plafond est «décoré d'une tapisserie, où l'on voit des insulaires des îles Sandwich, de la Société, des Amis, \&c. dans leur grand et brillant costume, exécutant des danses, des évolutions militaires, \&c (ce qui) ne contribue pas peu à augmenter le plaisir du spectateur» ${ }^{24}$.

Accompagnée de musique italienne, l'exposition est installée dans une salle divisée en trois sections par deux rideaux d'étoffe et est éclairée par deux lustres, deux lampes et deux chandeliers. De même, fidèle au modèle du cabinet, le Musée italien est doté d'une table servant à la consultation et à l'examen des objets et de trois bancs ${ }^{25}$, vraisemblablement destinés au confort des visiteurs.

Dans la première section, qui regroupe de loin le plus grand nombre d'objets, se retrouvent «des animaux et autres productions naturelles» et douze figures en cire grandeur nature. Soucieux de présenter au public la variété espérée par les amateurs d'histoire naturelle, Delvechio y regroupe «une collection choisie de Quadrupèdes, d'Amphibies, de Reptiles, d'Oiseaux et de Poissons, si bien empaillés et disposés, qu'on les croirait vivants, si on ne les voyait de près et au grand jour» ${ }^{26}$.

Parmi ces animaux figurent notamment, au compte des mammifères, un ours blanc, un léopard, une panthère, un lionceau, un jeune tigre, un jeune sanglier, deux porcs-épics, un «armadillo» (tatou), deux lynx, deux loups-cerviers, une loutre et deux yeux de baleine. Chez les reptiles, on retrouve trois lézards, un crocodile du Nil, un caméléon,

23 Le Spectateur canadien, 14 août 1824.

24 Bibaud, op. cit., 54.

25 Le Spectateur canadien, 14 août 1824; Bibaud, op. cit., 54; ANQ-M, Inventaire après décès de Thomas Delvechio, op. cit.

26 La Gazette de Québec, 17 août 1829; Bibaud, op. cit., 54-55. 
cinq serpents (dont le clotho ou vipère léthifère de l'Amérique du sud, la «broderie» et l'anaconda de Ceylan «très curieux par la couleur brillante de sa peau») et un flacon «rempli de différents serpents, conservés dans de l'esprit de vin». Les oiseaux y sont représentés par une autruche, un albatros, une grande poule de Russie, un toucan, un pélican, un ibis écarlate, un oiseau du paradis, des faisans, des perroquets, des oiseaux tropicaux «remarquables par la beauté et la variété de leur plumage», deux aigles et un œuf d'autruche; en tout, une collection ornithologique «au nombre de cinquante trois différentes espèces». Les poissons offerts à l'admiration du public sont un poisson-diable, un goulu de mer, un soleil et deux étoiles de mer, un poisson armé à scie double et «une autre espèce de poisson armé». Qui dit histoire naturelle au XIX ${ }^{e}$ siècle dit encore «Conques et Coquillages de mer» et le Musée italien en possède une collection «de différentes espèces», remarquables par leurs «couleurs et configurations». Finalement, on trouve dans cette section trois cadres «dont le fond est couvert de papillons secs» et une collection de plantes marines ${ }^{27}$.

Fait révélateur de la vocation curieuse du musée, le volet d'histoire naturelle n'occupe qu'une partie de la première section et voisine avec la collection de curiosités naturelles, liées aux spécimens d'histoire naturelle précédents, mais abordées comme lusus naturae: «un Agneau à huit jambes (...), une tête de Bélier à quatre cornes, un flacon avec un cochon à huit pattes et deux têtes». La section est complétée par un premier volet de curiosités artificielles, comprenant 10 figures de cire représentant entre autres la «belle albinesse», «Charlotte Corday poignardant Marat, un homme rasé par un singe, un écolier qui écrit», une famille péruvienne, les «beautés de Philadelphie, de Boston et de Montréal» et une figure en bois dont la nature exacte demeure inconnue ${ }^{28}$.

Dans les seconde et troisième sections, décrites de façon beaucoup plus brève dans l'ensemble des sources disponibles, se concentrent «les curiosités exclusivement artificielles», et particulièrement les appareils mécaniques. S'y trouvent d'abord cinq figures de cire automates «de jeunes garçons et de jeunes filles qui paraissent animées et jouent des airs sur des timbres». Viennent ensuite «une très-bonne optique pourvue de belles vues (nous y avons vu la ville de Londres comme on voit celle de Montréal de dessus la Montagne qui l'avoisine), un grand Concert mécanique d'Automates, et une Maison

27 Bibaud, op. cit., 54-55; Le Spectateur canadien, 14 août 1824; ANQ-M, Inventaire après décès de Thomas Delvechio, op. cit.

28 Idem. 
d'Industrie, l'un et l'autre supérieurs, à ce que nous croyons, à ce qui a jamais été vu en ce genre, au moins dans ce pays», douze «cadres avec des figures (...) un mécanisme complet, (...) deux électricités». Anecdote révélatrice du goût de Delvechio pour ce genre d'objets de curiosité, son inventaire après décès note au grenier de sa demeure une «petite maison pour les singes», des «effets d'une électricité», d'une «mélodie italienne» et de «trois boîtes métalliques pour la maison d'industrie», ce qui donne à penser que Delvechio procédait à un certain renouvellement de ses mécaniques et remisait celles qui n'étaient plus utilisables ou qu'il conservait peut-être des réserves pour son exposition. Dans une pièce séparée des trois sections de la salle principale, enfin, le visiteur peut voir des instruments de musique: une mélodie italienne et un jeu de cloches $^{29}$ dont les airs accompagnent la visite et qui semblent constituer en eux-mêmes des objets de curiosité.

Le contenu du Musée italien, typique du modèle américain des cabinets de curiosités, est donc hétéroclite et structuré autour d'une classification encore pré-scientifique. La collection d'histoire naturelle comprend, pour autant que l'on en puisse juger, une centaine de spécimens auxquels s'ajoutent un flacon contenant un nombre indéterminé mais certainement modeste de serpents et des collections vaguement décrites de coquillages, de papillons et de plantes. Elle est donc relativement limitée en termes d'objets et ne semble avoir que peu en commun avec celle de Pierre Chasseur, par exemple, qui ouvre à Québec en 1826 un cabinet d'histoire naturelle renfermant plus de 600 spécimens (dont seulement quelques curiosités) autour desquels le naturaliste amateur rassemble «tout ce qui tend à (les) caractériser, de manière à nous donner tout à la fois en quelque sorte l'histoire et les habitudes (des animaux) en vue» ${ }^{30}$. Quant à la collection de curiosités naturelles et artificielles de Delvechio, elle représente environ une trentaine d'objets, auxquels on peut ajouter ceux qu'il conserve au grenier. Moins considérable en quantité, elle couvre néanmoins plus des deux tiers de la surface d'exposition, ce qui laisse à penser que ces objets, généralement de plus grandes dimensions, formaient proba-

29 Bibaud, op. cit., 55; La Gazette de Québec, 17 août 1829; ANQ-M, Inventaire après décès de Thomas Delvechio, op. cit.

30 Lors du recensement de la collection de Chasseur effectué en 1836 par le Dr JeanBaptiste Meilleur pour le compte de l'Assemblée Législative du Bas-Canada, qui désire en faire l'acquisition, on énumère spécifiquement 606 spécimens d'histoire naturelle, dont 448 spécimens d'ornithologie, ainsi qu'une quantité non déterminée d'autres spécimens. Dans la catégorie des objets de curiosité, on ne compte que 6 artefacts. Voir Journal de la Chambre d'Assemblée du Bas-Canada, vol. 45, 22 février 1836, app. 0.0, 5e rapport du comité permanent sur l'éducation et les écoles; Michel Bibaud, «Zoologie du Bas-Canada», Bibliothèque canadienne, 2 (1826). 
blement une portion thématiquement aussi importante du musée que les spécimens d'histoire naturelle. Le fait que Bibaud accorde moins d'attention aux curiosités dans sa description du musée s'explique vraisemblablement par son intérêt personnel pour l'histoire naturelle et les sciences ${ }^{31}$. Estimée à 70 livres, $12 \mathrm{c}, 9 \mathrm{~d}$ à la mort de Delvechio, la collection ne représente par ailleurs qu'une infime portion des valeurs de son propriétaire, chiffrées au total à plus de 1159 livres $^{32}$. Si l'on en soustrait la mélodie italienne prisée à elle seule à 30 livres, l'essentiel de la collection vaut donc environ 40 livres, soit bien peu par rapport aux avoirs totaux de Delvechio, mais déjà plus que toute la fortune de bien des artisans de la même époque.

Delvechio offre donc aux visiteurs une très modeste collection et son Musée italien constitue en définitive une entreprise commerciale qui exploite le marché fertile de la curiosité et la sociabilité de la société anglaise. Il n'est associé à aucune organisation scientifique sérieuse, comme ce sera le cas du musée de la Natural History Society of Montreal en 1827. En ce sens, il est permis de placer sur le compte de l'enthousiasme pour la nouveauté et l'amusement scientifique, l'encensement auquel se livre Michel Bibaud concernant le potentiel didactique du Musée italien:

Nous conseillerions de le voir, non seulement aux personnes qui veulent se récréer agréablement, mais encore à celles qui ont quelque désir de s'instruire, persuadé que des visites à ce Cabinet leur vaudraient autant, et même mieux, que des leçons d'un professeur d'Histoire Naturelle, qui n'aurait pas les mêmes objets à mettre sous les yeux de ses élèves. ${ }^{33}$

\section{2 - LE PASSAGE DANS L'INDIFFÉRENCE}

Le Musée italien ne se révèle pas un succès commercial, malgré la vogue de la curiosité. De mai 1825 à janvier 1826, Delvechio doit rappeler aux Montréalais qu' «après plus de quatre années de dépenses et de soins, il a enfin formé et arrangé complètement son Cabinet de Curiosités Naturelles et Artificielles, et qu'il est maintenant prêt à être montré». Rappelant les vocations de son établissement, il «ôse se flatter que les amateurs de l'Histoire Naturelle, de l'Art, et de l'Harmonie, y trouveront de quoi satisfaire amplement leur curiosité» ${ }^{34}$.

31 Chartrand et al., op. cit., 94.

32 ANQ-M, Inventaire après décès de Thomas Delvechio, op. cit.

33 Bibaud, op. cit., 54.

34 Le Spectateur canadien, 28 mai 1825. 
Devant l'insuccès de cette démarche, il tente une dernière fois de mettre en valeur son entreprise et lance au public montréalais, de janvier à mai 1826, une menace de fermeture:

Le propriétaire du Musée d'Histoire naturelle et cabinet de curiosités, en faisant ses remerciements aux personnes qui ont bien voulu l'honorer de leur faveur, croit pouvoir dire que l'encouragement qu'il en a eu jusqu'ici ne lui a pas paru suffisant pour le dédommager de ses peines et de ses dépenses; et comme il lui a été offert de son Cabinet un prix qui lui a paru raisonnable, il est déterminé à s'en défaire à l'ouverture de la navigation, si l'encouragement ne devient pas plus considérable; si au contraire la faveur du public augmente, il se fera un devoir de lui conserver ce moyen d'amusement et de récréation, le seul du genre qu'il y ait dans le pays, quand même il trouverait des offres plus avantageuses que celles qui lui ont déjà été faites. [Delvechio reste même] disposé à le rendre plus considérable, si les circonstances le permettaient. ${ }^{35}$

Delvechio n'a toutefois pas le loisir de passer aux actes puisqu'il décède le 5 mai 1826. Il lègue par testament tous ses biens et propriétés à son épouse, Thérèse Chevalier, et ordonne que chacune de ses filles reçoive 500 livres à son mariage ${ }^{36}$. L'historiographie veut ensuite que son gendre, Pierre Cajetan Leblanc, aubergiste et épicier au marché neuf souvent présenté comme le «capitaine Leblanc», ait pris la relève du Musée italien, dont les collections auraient ultimement été acquises par la Natural History Society of Montreal ou le Jardin botanique Guilbault, ou encore auraient été dispersées en $1853^{37}$. Un examen attentif des sources révèle cependant un sort différent.

La veuve Delvechio continue en effet d'administrer le patrimoine familial, comme le démontrent les nombreux baux de location passés par elle devant notaire ${ }^{38}$. En 1828 , puis en 1832 , elle nomme Pierre Cajetan Leblanc exécuteur de ses affaires. Après le décès de sa bellemère en septembre 1832, celui-ci agira comme procureur des héritiers Delvechio lorsqu'il passera en leur nom un bail de location. Il administre enfin les biens de son épouse, malgré les dispositions du

35 Le Spectateur canadien, 21 janvier 1826.

36 ANQ-M, Registre d'état civil, Notre-Dame de Montréal, 8 mai 1826, n 86; ANQ-M, Testament de Thomas Delvechio, op. cit.

37 Dubé et Montpetit, op. cit., 12; Massicotte, op. cit., 127; Duchesne, «Delvecchio, Thomas», op. cit., 6: 202-204.

38 ANQ-M, baux de location de Thérèse Chevalier, greffe Nicolas-Benjamin Doucet, 10 avril 1827, $\mathrm{n}^{\circ} 14444 ; 14$ février 1828, $\mathrm{n}^{\circ} 13104 ; 15$ février 1830, $\mathrm{n}^{\circ} 17451 ; 22$ mai 1830, $\mathrm{n}^{\circ} 17521$. 
testament de Thomas Delvechio stipulant que ses enfants devaient en avoir la gestion et l'usufruit «sans le contrôle, participation ou autorisation de leurs époux» ${ }^{39}$. Toutefois, malgré cette main mise sur la succession de Delvechio, Leblanc ne relance pas le Musée italien, vraisemblablement toujours ouvert place du Vieux-marché. Probablement devenu un fardeau pour la veuve et sa famille, le Musée est mis en vente en septembre 1829 par la succession ${ }^{40}$ et acquis par le frère de Pierre, Joseph Cajetan Leblanc, épicier de Montréal. Celui-ci, qui y voit sans doute un bon investissement malgré les difficultés rencontrées par son fondateur, en annonce par la suite la réouverture en août 1830. Conscient de l'importance croissante des sciences naturelles dans la société montréalaise, qui a justifié en 1827 la création du musée de la Natural History Society of Montreal, il présente désormais le Musée italien comme cabinet d'histoire naturelle, abandonnant l'aspect de curiosité. En 1833, une annonce plus élaborée décrit la collection en reprenant l'essentiel de la formulation originelle de 1824 par Delvechio ${ }^{41}$. Entreprise commerciale vieillissante, le Musée italien ne renouvelle pas sa collection après 1826. Tout au plus y présente-t-on parfois des attractions populaires.

Comme Delvechio avant lui, Leblanc recommence en effet rapidement à tabler sur la curiosité pour amener des visiteurs au musée. De décembre 1834 à février 1835, reprenant la recette éprouvée des premiers temps de l'Auberge des Trois-Rois en 1791, il annonce dans La Minerve la tenue de «soirées amusantes». Il vient en effet «de mettre son Muséum à la disposition de M. Giovani, arrivé tout récemment de Paris, pour offrir au Public la représentation des Ombres Chinoises, la Lanterne Magique et d'autres Objets de Curiosités du Musée ${ }^{42}$. On peut penser qu'il s'agit là du dernier effort de publicité du propriétaire, qui semble dorénavant se concentrer sur son négoce d'épicier et sur ses nombreuses propriétés immobilières ${ }^{43}$. Associé à une épicerie, le Musée italien, sous Joseph Cajetan Leblanc, devient une composante banale du paysage culturel montréalais.

39 ANQ-M, greffe Nicolas-Benjamin Doucet, 19 mai 1828, $\mathrm{n}^{\circ}$ 15351; 8 février 1832, $\mathrm{n}^{\circ}$ 19340, 17 janvier 1833, $\mathrm{n}^{\circ}$ 20140; ANQ-M, acte de notoriété de Pierre Cajetan Leblanc, greffe Joseph-Hilarion Jobin, 4 avril 1842, n ${ }^{\circ} 3300$; ANQ-M, Testament de Thomas Delvechio, op. cit.

$40 \quad$ La Gazette de Québec, 17 août 1829.

41 La Minerve, 19 août et 4 octobre 1830; 8 juillet et 26 septembre 1833.

42 La Minerve, 8 décembre 1834.

43 ANQ-M, baux de location par Joseph Cajetan Leblanc, greffe John Blackwood, 29 avril $1834, \mathrm{n}^{\circ} 268 ; 11$ février $1845, \mathrm{n}^{\circ} 85$. Greffe Joseph-Hilarion Jobin, 28 janvier 1842, $\mathrm{n}^{\circ} 3166 ; 12$ décembre $1842, \mathrm{n}^{\circ} 3213 ; 10$ janvier $1845, \mathrm{n}^{\circ} 4704 ; 28$ octobre $1846, \mathrm{n}^{\circ} 5571$. Greffe Joseph Belle, 8 mai $1845, n^{\circ} 8623$. 
En 1833, le musée déménage au 37 de la rue Saint-Paul, puis une seconde fois, entre 1834 et 1842 , au 107 de la même rue, comme l'attestent les différentes éditions du Montreal Directory de 1842 à 1845. On le retrouve alors désigné comme «Leblanc, J.C., grocery and museum, $107 \mathrm{St}$-Paul street» ${ }^{44}$. En juin 1847, Leblanc décède et laisse par testament ses avoirs à son épouse Louise Rollin et à ses enfants. Dès le mois d'août, ses héritiers vendent à l'encan le musée, dépassé par des institutions plus récentes et plus savantes - notamment le musée de la Natural History Society of Montreal - et toute sa «belle collection d'objets rares et curieux», dont on imprime un catalogue ${ }^{45}$. Les objets sont alors achetés séparément et la collection définitivement dispersée. En 1852, Germain Cajetan Leblanc, frère de Joseph et Pierre, installe sa taverne dans les anciens locaux du musée au 107 de la rue Saint-Paul, puis les quitte l'année suivante pour un emplacement rue $\mathrm{Craig}^{46}$.

\section{3 - LE MUSÉE ITALIEN ET LA MONTÉE DES SCIENCES}

À mesure que l'on avance dans le siècle, les institutions scientifiques prennent une ampleur nouvelle au sein de la société canadienne et laissent de moins en moins d'espace aux formes populaires de la curiosité. Le passage à Montréal et à Québec en 1840 du philanthrope français Alexandre Vattemare, que seul le climat politique empêche d'instaurer un système d'échange de spécimens d'histoire naturelle et de livres ainsi qu'un institut regroupant sous un même toit les principales institutions culturelles des deux villes, amorce une sensibilisation des élites sociales à la nécessité de réformer les lieux de savoir. Le développement, dans la seconde moitié du siècle, du système d'éducation reposant sur les collèges classiques entraîne dans plusieurs de ces institutions la création de musées d'histoire naturelle $^{47}$. La Commission géologique du Canada, créée en 1842, s'installe à Montréal et y ouvre elle aussi un musée. Les musées déjà existants, quant à eux, se professionnalisent: à Montréal, par exemple, si le musée de la Natural History Society of Montreal possède, dès son ouverture en 1827, une certaine quantité de curiosités, le registre des

44 Montreal Directory, 1842-1843 à 1845-1846.

45 ANQ-M, Testament de Joseph Cajetan Leblanc, greffe Joseph-Hilarion Jobin, 30 janvier 1847, $\mathrm{n}^{\circ}$ 5622.; La Revue canadienne, 13 août 1847.

46 Montreal Directory (1852): 145; (1853): 152.

47 Élizabeth Revai, «Le voyage d'Alexandre Vattemare au Canada: 1840-1841. Un aperçu des relations culturelles franco-canadiennes: 1840-1857», Revue d' histoire de l'Amérique française, 22,2 (septembre 1968): 257-299; Claude Galarneau, Les collèges classiques au Canada français (1620-1970) (Montréal, Fides, 1978), 25-43. 
acquisitions de 1827 à 1853 confirme leur rapide abandon au fil des ans et la concentration exclusive du musée sur l'histoire naturelle ${ }^{48}$. L'objet, inséré dans une démarche scientifique, cesse graduellement d'être une curiosité individuelle pour devenir partie significative d'un tout, d'une typologie. Dans ce nouvel ordre de choses, le modèle du cabinet de curiosités naturelles et artificielles ne trouve plus sa place. La démarcation se précise entre le musée «savant» et la curiositéspectacle, celle-ci devenant peu à peu l'exclusivité des expositions itinérantes et fêtes foraines qui offriront avec éclat au public, jusqu'à la fin du siècle, des collections de curiosités (les «dime museums») similaires à celles des cabinets ${ }^{49}$.

Le Musée italien, premier musée à vocation permanente de Montréal, procède en droite ligne du modèle américain du cabinet de curiosités et offre au public une collection constituée de façon à exploiter les goûts de l'époque. N'ayant de lien avec aucune société savante et procédant davantage du monde de la curiosité-spectacle à l'américaine que de celui des sciences, il ne correspond pas à la définition nouvelle du musée savant où l'histoire naturelle est sujet d'étude plutôt qu'objet d'amusement. Accueilli par une relative indifférence, il disparaît au moment où s'établit entre musée et spectacle une distinction que, par un ironique retour des choses, nos musées d'aujourd'hui ont entrepris de réviser au profit du divertissement.

48 Bibliothèque canadienne, 6,6 (mai 1828): 235-236; McGill University, BlackerWood Library, Registry of Miscellaneous Donations to the Museum (1827-1853), ms.

49 Alexander, Museum Masters, op. cit., 68-69; Carle et Duchesne, «L'ordre des choses...», op. cit., 13-23. 